\title{
Aneurismas de la región de la arteria oftálmica
}

\author{
S.D. Vega-Basulto; F.G. Gutiérrez-Muñoz; G. Mosquera-Betancourt; F. Rivero-Truit y S. A. Vega-Trenado
}

Servicio de Neurocirugía. Hospital Manuel Ascunce Doménech. Camagüey. Cuba

Resumen

Introducción. Los aneurismas de la arteria oftálmica son lesiones poco frecuentes $y$ con particularidades específicas.

Objetivo. Presentar las características anatomoclínicas, quirúrgicas y los resultados del tratamiento microquirúrgico de nuestros pacientes con aneurismas de esta región.

Pacientes y métodos. 604 pacientes con aneurismas intracraneales fueron llevados al quirófano entre enero de 1982 y diciembre del 2004. Hubo 50 pacientes con aneurismas de la región de la arteria oftálmica. El promedio de edad fue de 52 años, el $80 \%$ fueron mujeres, el $60 \%$ presentaban aneurismas múltiples y un $20 \%$ gigantes. El 12\% poseían lesiones aneurismáticas bilaterales. El $20 \%$ de los aneurismas fueron incidentales. El $\mathbf{5 0 \%}$ tenían manifestaciones oftalmológicas y la frecuencia se elevó al $\mathbf{1 0 0 \%}$ en los aneurismas grandes y gigantes. El $16 \%$ de los trastornos visuales fueron ignorados o confundidos inicialmente. En aneurismas múltiples empleamos la ruta pterional uni o bilateral asociada a otros abordajes. En los aneurismas oftálmicos grandes o gigantes utilizamos la técnica de descompresión-succión retrógrada modificada.

Resultados. Treinta pacientes $(60 \%)$ obtuvieron una recuperación completa, catorce pacientes $(\mathbf{2 8 \%})$ una recuperación incompleta y dos incapacidad grave. No hubo pacientes en estado vegetativo. Hubo cuatro fallecidos (8\%). El $\mathbf{5 0 \%}$ de los déficit postoperatorios desaparecieron y el $64 \%$ de los trastornos visuales mejoraron a los tres meses.

Conclusión. El método microquirúrgico permitió tratar aneurismas de la región de la arteria oftálmica únicos, múltiples, bilaterales, grandes y gigantes con buena recuperación de los déficit neurológicos y de los trastornos visuales.

PALABRAS CLAVE: Aneurismas intracraneales. Aneu-

Recibido; 06-06-05. Aceptado: 22-11-05 rismas de la arteria oftálmica. Arteria carótida interna. Aneurismas gigantes. Técnica de descompresión succión retrógrada.

Ophthalmic artery aneurysms

Summary

Introduction. Ophthalmic artery aneurysms are infrequent lesions and they have very specific particularities.

Objective. to present the anatomoclinical and surgical characteristic of our patients with ophthalmic aneurysms and to analyze our surgical results.

Patient and methods. 604 patients with intracranial aneurysms were operated on between January 1982 and December 2004. There were 50 patients with ophthalmic artery aneurysms. Average age was 52 years, $80 \%$ were women, $60 \%$ harbored multiple aneurysms and $\mathbf{2 0 \%}$ were giant lesions. $12 \%$ had bilateral ophthalmic aneurysms. $20 \%$ of ophthalmic aneurysms were incidental. $\mathbf{5 0 \%}$ had ophthalmological manifestations; howewer, this presentation occurred in $100 \%$ of the patients with large and giant aneurysms. Initially, $12 \%$ of ophthalmologic disorders were missed. We utilized unilateral or bilateral pterional approach associated to others in multiple aneurysms. We performed retrograde decompression-suction technique modified for large and giant aneurysms.

Results. Thirty patients $(60 \%)$ obtained a complete recovery, fourteen patients $(28 \%)$ an incomplete recovery and two were seriously disabled. There were not patients in vegetative state. There were four deaths $(8 \%) .50 \%$ of postoperatory deficits disappeared and $64 \%$ of ophthalmologic disorders improved three months later.

Conclusion. Microsurgical method allowed to treat unique, multiple, bilateral, large and giant ophthalmic aneurysms with good recovery of neurological and visual deficits. 
KEY WORDS: Intracranial aneurysms. Ophthalmic aneurysms. Internal carotid artery. Giant aneurysms. Retrograde decompression-suction technique.

\section{Introducción}

La primera descripción de los aneurismas de la región de la arteria oftálmica, un subgrupo específico de aneurismas intracraneales, la hicieron Drake et $\mathrm{a}{ }^{13} \mathrm{y}$ las observaciones y reflexiones iniciales de estos autores permanecen cada día con más vigencia.

Los aneurismas de esta localización se originan en la pared medial o anteromedial de la arteria carótida interna, en el segmento entre la arteria oftálmica y el origen de la arteria comunicante posterior y ponen en peligro la vida del paciente debido al elevado riesgo de hemorragia intracraneal, compresión de estructuras neurales o ambas $^{8,25,37,46,51}$.

La experiencia acumulada con posterioridad al primer estudio $^{13}$ demostró que estas lesiones tienen algunas características muy particulares, que van mucho más allá de su simple localización anatómica y la proximidad con la arteria oftálmica.

La íntima relación de estos aneurismas con los nervios ópticos adyacentes, que cubren y tienden a ocultar su origen, la vecindad de las estructuras óseas de la base craneal y el seno cavernoso, así como las dificultades que se pueden presentar para clipar el cuello de estos aneurismas, preservando la integridad de la arteria madre, dan origen a complejidades técnicas muy especiales durante el tratamiento quirúrgico ${ }^{25,62}$.

La proximidad con la vía visual también determina anormalidades clínicas y rasgos muy significativos relacionados con la detección precoz de estas lesiones, con la manipulación microquirúrgica, con la recuperación visual postoperatoria y el pronóstico a largo plazo de estos pacientes ${ }^{15,62}$.

En este informe presentamos las características anatomoclínicas y quirúrgicas de un grupo de pacientes con aneurismas del segmento oftálmico y analizamos sus resultados generales y oftalmológicos.

\section{Pacientes y métodos}

Desde enero de 1982 hasta diciembre del 2004 fueron llevados al quirófano del Hospital "Manuel Ascunce Domenech" de Camagüey, Cuba, un total de 604 pacientes con aneurismas intracraneales. Las lesiones fueron confirmadas por evaluación clínica, estudios angiográficos completos y los hallazgos operatorios. Fueron excluidos los pacientes incompletamente estudiados, los que tenían lesiones total o parcialmente extradurales y los que no se sometieron a tratamiento quirúrgico o fallecieron antes de ir al quirófano.
Fueron seleccionados los pacientes con uno o más sacos aneurismáticos en la carótida, en la región de la arteria oftálmica, fueran sintomáticos o no. Para evaluar estas lesiones utilizamos la clasificación de Hoh et $\mathrm{al}^{25}$.

El estado clínico de los pacientes que ingresaron con hemorragia subaracnoidea fue evaluado preoperatoriamente con la Escala de la Federación Mundial de Neurocirujanos ${ }^{12}$ y la intervención quirúrgica se realizó cuando se hallaban en grado I ó II de esa escala. Los pacientes con grado III, IV y V ingresaron inicialmente, en la sala de Terapia Intensiva, para mejorar lo más rápido posible su estado clínico y lograr condiciones más favorables durante la cirugía.

En casos de multiplicidad aneurismática aplicamos el Algoritmo de Nehls y cols ${ }^{42}$.

El tamaño de los aneurismas fue clasificado con el mismo criterio del Estudio Cooperativo de Aneurismas de 1990 que consideró como pequeñas a las lesiones menores de $12 \mathrm{~mm}$, grandes los aneurismas entre 13 y $25 \mathrm{~mm}$ y gigantes los aneurismas mayores de $25 \mathrm{~mm}^{34,35}$.

Las anormalidades de la vía visual fueron evaluadas en cada paciente teniendo en cuenta su estado clínico neurológico y la forma de presentación. Se evaluaron al ingreso: agudeza visual, fondo de ojo y anomalías de los campos visuales. El campo visual fue evaluado, inicialmente, en la cama del paciente con la técnica de Trobe y Glaser $^{55}$. Los exámenes campimétricos subsiguientes a la intervención se realizaron en el laboratorio de oftalmología.

Se repitieron estudios de la agudeza visual, fondo de ojo y campimetría al egreso, tres y seis meses después del alta.

En relación con la terapéutica quirúrgica, los pacientes fueron clasificados en cuatro grupos. El grupo 1 incluyó a los pacientes con aneurismas únicos de la región carótido-oftálmica. El grupo 2 a los pacientes con aneurismas de la región carótido-oftálmica y del sistema carotídeo homolateral. El grupo 3 incluyó a los pacientes con lesiones aneurismáticas bilaterales y el grupo 4 cuando existían lesiones aneurismáticas asociadas en la región infratentorial.

Un total de 20 pacientes $(40 \%)$ tenían lesiones únicas (Tipo 1), 17 pacientes tenían aneurismas múltiples unilaterales (Tipo 2), 11 pacientes poseían lesiones múltiples bilaterales (tipo 3) y dos lesiones supra e infratentoriales (Tipo 4).

Las intervenciones quirúrgicas fueron realizadas por el autor del trabajo o bajo su supervisión directa. Se utilizaron soporte craneal de Sugita, neuroanestesia especializada, métodos microneuroquirúrgicos, clips de forma variada y seguimiento postoperatorio en la Unidad de Terapia Intensiva. No se utilizaron métodos endovasculares solos o combinados.

El abordaje pterional unilateral o bilateral ${ }^{62}$ fue utilizado 
en toda la serie. En todos los pacientes se realizó resección ósea alrededor del cuello aneurismático (canal óptico, apófisis clinoides y tubérculo selar según las necesidades). En los pacientes que requirieron una segunda intervención para tratar otros aneurismas, ésta se realizó dos a cuatro semanas después de la primera, en dependencia del estado clínico del paciente y su recuperación. La primera intervención siempre incluyó el aneurisma sintomático.

El 75\% de los pacientes fueron llevados al quirófano durante la primera semana después del sangrado y el $25 \%$ restantes antes de la segunda semana.

En los pacientes con aneurismas carótido-oftálmicos grandes y gigantes, la pared es gruesa, fibrótica e irregular, por lo que utilizamos siempre la técnica de descompresión-succión retrógrada descrita por Batjer y $\operatorname{cols}^{3,4}$ en 1990 y modificada por nosotros ${ }^{61}$. Esta técnica incluye, adicionalmente, una posición que facilite el acceso a los vasos del cuello y se monitorizó la función cardiorrespiratoria y el electroencefalograma intraoperatorio. Se utilizó manitol endovenoso en todos los casos para lograr un cerebro flácido y de fácil manejo. No se utilizaron rutinariamente el drenaje de LCR, la hipotensión arterial controlada ni procederes derivativos o cócteles antiisquémicos.

Realizamos protección cerebral con thiopental o etomidato e hipotermia leve.

La monitorización electroencefalográfica intraoperatoria se realizó con electrodos de aguja debidamente colocados en el cuero cabelludo, que no se desplazan con la manipulación de la cabeza ni con la humedad del suero de irrigación.

Utilizamos el thiopental o el etomidato para reducir el metabolismo cerebral al máximo, lo cual se corresponde con el silencio eléctrico cerebral registrado en el electroencefalógrafo. La temperatura corporal se permitió que descendiera hasta 32 a 34 grados centígrados, a través de la exposición del paciente sin mantas, deliberadamente, a la temperatura ambiental de la sala de operaciones.

Antes de comenzar la craneotomía pterional, la arteria carótida común se disecó en el cuello hasta su bifurcación, lo que expuso un segmento de la arteria carótida externa y otro de la interna y permitió observar el estado de la pared vascular.

El procedimiento quirúrgico de descompresión succión retrógrada modificado consistió en comprimir con clips, transitoriamente, las arterias carótida común, externa e interna proximal y distal al saco, previo a la emergencia de la arteria comunicante posterior. Esto conforma un área de atrapamiento vascular amplio cuyo contenido se vacía al introducir en la carótida interna un angiocatéter nro. 18. Este catéter se conecta a una llave de paso metálica y ésta, a su vez, a un aspirador Frazier 7, que, acoplado al sistema de aspiración de la sala de operaciones, succiona retrógra- damente la sangre contenida en el segmento antes descrito, colapsa sorprendentemente los gruesos vasos sanguíneos y "desinfla" el saco aneurismático.

Si este efecto no se logra, debe sospecharse algún fallo técnico en el procedimiento y debe revisarse completamente o pensar en la posibilidad de una trombosis parcial del saco aneurismático.

Estos pasos técnicos del atrapamiento y vaciado regional deben concebirse bien por cada uno de los miembros del equipo quirúrgico que ayudan al cirujano, de forma que cada uno conozca que tarea le corresponde y en que momento entra a desempeñar su función. En casos de equipos con experiencia previa, esto tiene poco significado, pero cuando se ejecuta por primera vez o por equipos con poco entrenamiento, debe ensayarse antes de comenzar la operación. Hemos observado que el desajuste de los pasos y su falta de orden determina pérdida de tiempo, prolongación de la isquemia y accidentes embólicos.

El clipaje temporal se realizó con clips diseñados por Yasargil (Aesculap) para esos efectos. El tiempo de oclusión osciló desde 3 hasta 16 minutos y se utilizó para la disección del vaso madre, el cuello del aneurisma y los afluentes. En los pacientes donde esta maniobra se prolongó, el tiempo de oclusión se repartió en dos o tres ocasiones para permitir un tiempo prudencial de reperfusión entre una y otra maniobra. Un paciente requirió de tres oclusiones, aunque generalmente se utilizó una sola.

Después del clipaje definitivo, el saco se puncionó con una aguja calibre 26 para evacuar totalmente su contenido y confirmar la completa oclusión del aneurisma. En pacientes con sacos muy voluminosos y gruesos, estos se resecaron, siempre que fuera técnicamente posible y no añadiera un riesgo adicional.

Resultó de gran ayuda, antes del clipaje, la observación del estado anatómico de la pared aneurismática. Paredes normales con áreas de afinamiento, áreas de ateromatosis próxima al cuello y la presencia de "mamelones" en la pared modifican la actitud del cirujano en cada paso. Las áreas de afinamiento resultaron peligrosas para la coagulación bipolar debido a la rotura potencial, mientras que las áreas de ateromatosis del vaso madre o próximas al cuello hicieron el clipaje transitorio peligroso por el riesgo de fractura de la pared arterial o de embolismo distal de un fragmento de placa ateromatosa.

Utilizamos siempre normotensión arterial y tuvimos en cuenta que los barbitúricos hacen descender la presión arterial intraoperatoria. El flujo sanguíneo se restableció completamente una vez completado el clipaje. La maniobra de reperfusión se hizo con la retirada del primero de los clips de la carótida común, luego la externa y por último la interna. Primero retiramos el clip en la carótida interna distal y, por último, el proximal, lo cual restablece muy lentamente la presión sobre la grapa. Este es el momento 
de confirmar la completa oclusión del cuello o de detectar un deslizamiento del clip. En casos de duda, el angiocatéter colocado en la arteria carótida interna en el cuello, puede utilizarse para realizar una arteriografía intraoperatoria. Este catéter requiere que se preste una atención máxima en su introducción y manipulación, ya que puede dañar la pared arterial, desprender placas de ateromas, producir disecciones de la pared o formar émbolos que emigran a la arteria cerebral media durante la reperfusión; por ello, nosotros siempre utilizamos pequeñas dosis de suero fisiológico con heparina para lavar y mantener lleno el angiocatéter y la llave de paso y para realizar una irrigación suave del área de atrapamiento.

No utilizamos la circulación extracorpórea con parada circulatoria en ninguno de los pacientes.

En los aneurismas próximos al origen de la arteria oftálmica, la reseción ósea facilitó colocar el clip de forma tal que las mandíbulas de la grapa se cierren paralelamente al eje mayor de la pared de la arteria madre, de lo proximal a lo distal (anterógradamente).

Los aneurismas de la arteria hipofisaria superior desplazan el nervio óptico superiormente y la arteria carótida superior o superolateralmente. La extensión proximal del aneurisma se confina al piso de la base craneal, debajo del nervio óptico. La clave de la disección estuvo en definir la extensión del cuello tanto proximal como distalmente. Los clips fenestrados, que incluyen la carótida en el anillo, colocados en tandem, bien alineados, aplicados en dirección de distal a proximal y siguiendo el eje central de la carótida, ayudaron a reconstruir la arteria. Los clips avanzaron hasta lograr contacto óseo debajo de la pared del nervio óptico. Alternativamente, pudo aplicarse un clip convencional, aunque anatómicamente pueda parecer contradictorio, ya que con un adecuado vaciado del saco, una reconstrucción de lo medial a lo lateral fue una solución excelente.

Todas las maniobras quirúrgicas auxiliares tuvieron como objetivo localizar el cuello aneurismático y tener control del vaso madre proximalmente, reducir el flujo sanguíneo retrógrado a través de la arteria oftálmica o ramas intracavernosas que mantienen la turgencia del aneurisma y su tensión sobre la vía óptica y desplazan el clip que se coloca en el cuello.

Los pacientes se atendieron en la Unidad de Terapia Intensiva por personal médico entrenado en el manejo postoperatorio en neurocirugía vascular. El uso de barbitúricos determina que el paciente demore en recuperarse; pero vigilamos la posible depresión respiratoria o la hipotensión postoperatoria, ya que ambas tienen un efecto perjudicial notable. Usamos manitol endovenoso en bajas dosis para mejorar la reología y la perfusión cerebral.

Los resultados postoperatorios se evaluaron con la Escala de Pronóstico de Glasgow ${ }^{32}$ seis meses después de la intervención quirúrgica. Esta escala considera cinco resultados: recuperación completa, incapacidad parcial, incapacidad grave, estado vegetativo y fallecido.

Tabla 1

Características clínicas generales de los pacientes

Edad
Media
52 años
Mayores de 60 años
$8(16 \%)$

Sexo
Mujeres
$40(80 \%)$
Hombres
$10(20 \%)$

Frecuencia general

$8.3 \%$

Aneurismas oftálmicos sintomáticos

$40(80 \%)$

Hemorragia

$25(50 \%)$

Hemorragia/compresión

$13(26 \%$

Compresión vía óptica

$12(24 \%)$

Aneurismas oftálmicos incidentales

$10(20 \%)$

Multiplicidad aneurismática

$30(60 \%)$ 
Tabla 2

Localización de los sacos aneurismáticos

\begin{tabular}{lc}
\hline Localización & Nro \\
\hline Aneurismas de la oftálmica unilateral & 44 \\
a) Únicos & 20 \\
b) Asociados a otras localizaciones & 24 \\
Aneurismas de la comunicante posterior & 12 \\
Aneurismas de la comunicante anterior & 10 \\
Aneurismas de la arteria cerebral media & 9 \\
Aneurismas de la oftálmica bilaterales & (12 sacos) \\
Aneurismas de la bifurcación carotídea & 2 \\
Aneurismas de la cerebral anterior distal & 2 \\
Aneurismas de la cerebelosa posteroinferior & 2 \\
\hline Total & $\mathbf{9 3}$ \\
\hline
\end{tabular}

\section{Resultados}

Encontramos 50 pacientes que tenían uno o más sacos aneurismáticos en la carótida en la región de la arteria oftálmica, los que constituyeron el $8.3 \%$ del total. Uno de ellos tenía un adenoma pituitario no funcionante que coexistía con un aneurisma de esa región.

\section{Características clínicas}

La edad promedio de la serie fue de 52 años. Treinta y cinco pacientes $(70 \%)$ se encontraban en la sexta década de la vida y ocho $(16 \%)$ eran mayores de 60 años. Cuarenta $(80 \%)$ eran mujeres y $10(20 \%)$ eran hombres (Tabla 1$) .40$ pacientes $(80 \%)$ tenían sacos aneurismáticos sintomáticos de la región de la oftálmica. Las manifestaciones clínicas principales fueron el sangrado en 25 pacientes $(50 \%)$ y la compresión de la vía óptica en 12 (24\%). En 13 pacientes (26\%) hubo ambas manifestaciones. En 10 pacientes $(20 \%)$ los aneurismas carótido-oftálmicos fueron incidentales y la causa de los síntomas fueron aneurismas de otra localización.

Todos los pacientes con hemorragia subaracnoidea seleccionados para cirugía se encontraban en el grado I o II de la Escala de la Federación Mundial de Neurocirujanos.

\section{Multiplicidad, localización y tamaño de las lesiones}

En 30 pacientes $(60 \%)$ se demostró más de un aneurisma. Hubo 93 sacos aneurismáticos en total. Treinta y siete estaban en otras localizaciones (Tabla 2) y las más frecuentes fueron las regiones de la arteria comunicante posterior y de la comunicante anterior. El promedio de sacos por paciente con aneurismas múltiples fue de $2.4 \mathrm{y}$

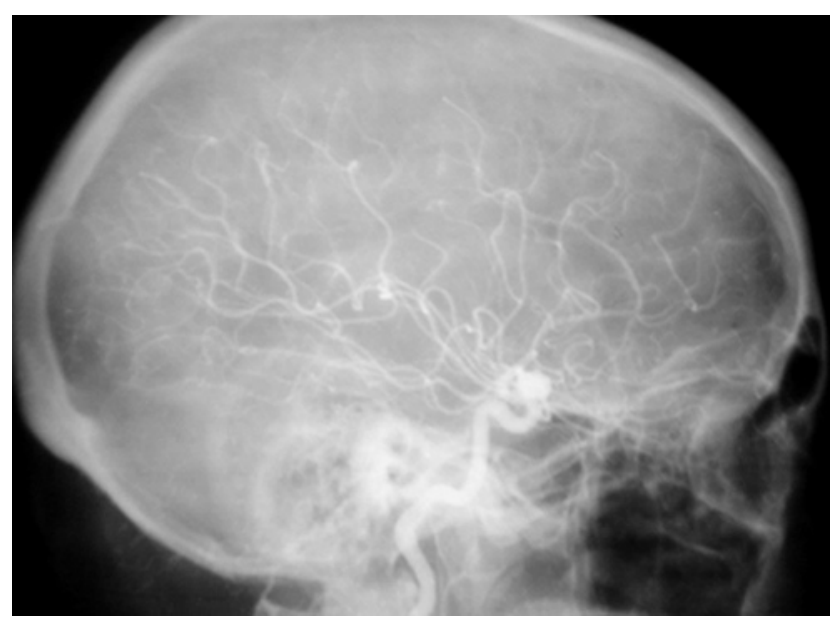

Figura 1. Angiografía carotídea izquierda. Vista lateral. Se observa un aneurisma sacular pequeño de la carótida en la región de la arteria oftálmica.

en siete se detectaron más de dos lesiones. En seis (12\%) existieron aneurismas bilaterales (gemelos o en espejo) en la región de la arteria oftálmica y dos pacientes (4\%) tenían lesiones aneurismáticas infratentoriales (arteria vertebral en la región de la arteria cerebelosa posteroinferior).

El tamaño de los aneurismas clasificado con el criterio del Estudio Cooperativo de Aneurismas de 1990 34,35 evidenció que $36(72 \%)$ eran pequeños (Figura 1), cuatro grandes $(8 \%)$ y diez $(20 \%)$ gigantes. De las treinta y siete lesiones asociadas 35 eran pequeñas y dos grandes. No hubo aneurismas gigantes asociados. Todos los aneurismas grandes o gigantes de la región de la arteria oftálmica fueron sintomáticos. Todos los aneurismas oftálmicos incidentales fueron pequeños y cuatro de ellos medían $4 \mathrm{~mm}$.

Hubo 56 aneurismas en la región de la arteria oftálmica. Cuarenta y siete (83\%) estaban ubicados en el origen de la arteria oftálmica y nueve (17\%) en el origen de la hipofisaria superior. Los aneurismas carótido-cava y transicionales (componente extradural) fueron excluidos ${ }^{25}$.

\section{Trastornos de la vía visual}

Las manifestaciones oftalmológicas detectadas fueron: deterioro de la visión monocular homolateral a la lesión aneurismática, trastornos campimétricos uni o bilaterales y palidez papilar revelada en el fondo de ojo y hemorragias subhialiodeas. Las alteraciones de la vía óptica se observaron en 25 pacientes $(50 \%)$. El deterioro de la visión previo al sangrado subaracnoideo se relacionó, injustificadamente, en ocho pacientes (16\%) a la edad avanzada, presbicia, glaucoma, catarata senil, ambliopías u otros trastornos retinianos. Las manifestaciones visuales estuvieron presentes en todos los pacientes con aneurismas grandes o gigantes de la región de la oftálmica y sólo en 11 de los pacientes 
Tabla 3

Manifestaciones oftalmológicas en aneurismas de la región de la arteria oftálmica

\section{Manifestación}

Deterioro de la agudeza visual

$25(50 \%)$

Trastornos campimétricos

Unilateral

$16(32 \%)$

Bilateral

$6(12 \%)$

Palidez papilar

Unilateral

$14(28 \%)$

Bilateral

Trastornos visuales en aneurismas grandes o gigantes

$2(4 \%$

$15 / 15$

Trastornos visuales en aneurismas pequeños con aneurismas pequeños. (Tabla 3 ).

Al ingreso, los trastornos del campo visual fueron unilaterales en 22 pacientes: en 16 (32\%) estaba involucrada inicialmente la región nasal homolateral inferior, en dos se observó un déficit altitudinal inferior y en cuatro la visión estuvo restringida sólo a la percepción de la luz en el ojo afectado. Dos pacientes con aneurismas gigantes de la arteria hipofisaria superior exhibieron una pérdida ligera del campo temporal superior y dos tuvieron déficit visuales bilaterales: uno bitemporal y otro homónimo.

Hubo palidez papilar en 16 pacientes: en 14 homolateral a la lesión y en dos bilateral, relacionados con lesiones gigantes de la hipofisaria superior.

En los pacientes con manifestaciones de sangrado y compresión de la vía visual, estas últimas fueron unilaterales en siete pacientes y bilateral en uno (hemianopsia bitemporal). Hubo un paciente con síndrome de Terson $(2 \%)$ que tenía un aneurisma pequeño roto en la región de la arteria oftálmica.

\section{Procederes quirúrgicos}

En 42 pacientes se utilizó un proceder quirúrgico (84\%) y en ocho, dos procederes (16\%) por aneurismas supratentoriales bilaterales o suprainfratentoriales (tipo 3 y 4). Se emplearon dos procederes en: cuatro pacientes con sacos que presentaban complejidades técnicas ubicados en la región de la arteria comunicante posterior contralateral, en otros dos pacientes con lesiones de la bifurcación de la arteria cerebral media contralateral y en dos con un saco aneurismático de la vertebral en el origen de la arteria cerebelosa posteroinferior; en ambos se realizó una craneotomía suboccipital extralateral.
En los seis pacientes con lesiones aneurismáticas bilaterales de la arteria oftálmica (aneurismas gemelos o en espejo) se utilizó un proceder quirúrgico. La lesión aneurismática contralateral a la vía de abordaje siempre fue pequeña y se ubicó en la propia región de la arteria oftálmica. No hubo lesiones contralaterales en los aneurismas de la región de la hipofisaria superior.

Se cliparon un total de 84 lesiones, se reforzaron ocho aneurismas pequeños menores de $4 \mathrm{~mm}$ y se realizó una aneurismorrafia.

\section{Resultados después de la cirugía}

Los resultados quirúrgicos generales se evaluaron a los seis meses a través de la Escala de Pronóstico de Glasgow y se evidenció que treinta pacientes $(60 \%)$ tenían una recuperación completa, catorce pacientes $(28 \%)$ una recuperación incompleta y dos incapacidad grave. No hubo pacientes en estado vegetativo.

Hubo cuatro fallecidos en la serie (8\%). Dos tenían aneurismas gigantes extremadamente voluminosos y otros dos tenían aneurismas múltiples. Uno de los pacientes con un aneurisma gigante falleció por un infarto en el territorio carotídeo homolateral a la zona del clipaje. El otro tenía una lesión muy voluminosa, que no podía cliparse y se realizó resección total del saco y aneurismorrafia; pero el paciente falleció después de la intervención quirúrgica por un infarto carotídeo.

Los otros dos fallecidos tenían hemorragia subaracnoidea y lesiones aneurismáticas múltiples. Ambos presentaron vasoespasmo postoperatorio que no mejoró con ninguna terapéutica.

Los cuatro fallecidos fueron pacientes con manifes- 
taciones clínicas de hemorragia subaracnoidea. No hubo fallecidos en el grupo con manifestaciones de compresión neural.

Las principales manifestaciones postoperatorias fueron: déficit motor, trastornos neuropsíquicos, trastornos de la vía visual y diabetes insípida. Otras complicaciones como fístulas de LCR, sepsis regionales e hidrocefalia tuvieron una representación insignificante en número. No hubo hematomas intracraneales postoperatorios.

Los déficit motores y los trastornos neuropsíquicos aparecieron al egreso en el 38\% de los pacientes; pero el 50\% de esas manifestaciones desaparecieron seis meses después de la intervención. Estos tipos de trastornos postoperatorios fueron más frecuentes en las lesiones aneurismáticas grandes o gigantes.

De los 25 pacientes con trastornos visuales hubo mejoría en $16(64 \%)$ después del clipaje y descompresión del aneurisma, aunque existiera palidez papilar por atrofia óptica previa. La mejoría clínica fue menos frecuente en pacientes con aneurismas grandes o gigantes y en aquéllos con manifestaciones visuales de más de seis meses de duración. La multiplicidad aneurismática no influyó en los resultados obtenidos con los trastornos visuales.

El patrón general de mejoría de los trastornos oftalmológicos fue muy variable en nuestra serie. Predominó la recuperación de curso lentamente progresivo. Observamos tres pacientes con mejoría hasta un año después de la intervención. No se observaron restablecimientos dramáticos de la visión. La reducción del efecto de masa del aneurisma se relacionó, generalmente, con mejoría de las manifestaciones ópticas unos meses más tarde.

Tres de los cuatro pacientes con visión a la luz antes de la intervención quirúrgica no obtuvieron ninguna mejoría. Las manifestaciones oftalmológicas de este subgrupo de pacientes tenían años de evolución y todos habían sido evaluados por especialistas antes del ingreso, en varias ocasiones, en las cuales se había propuesto otro diagnóstico.

Los trastornos campimétricos y la atrofia óptica mejoraron parcialmente en todos los pacientes.

No observamos deterioro visual postoperatorio en ninguno de nuestros pacientes.

En tres pacientes con aneurismas grandes o gigantes de la región de la arteria hipofisaria superior, en estrecha relación con el tallo hipofisario, se observó diabetes insípida después de la intervención quirúrgica. Hubo una respuesta muy favorable a la terapéutica hormonal sustitutiva y las manifestaciones clínicas fueron reversibles en todos los pacientes pasados seis meses de la intervención. En el paciente con adenoma hipofisario no secretor se asociaron manifestaciones de déficit hormonal de la hipófisis y se aplicó tratamiento sustitutivo de todos los ejes hormonales incluyendo la hormona antidiurética.

\section{Discusión}

Drake et $a^{13}$ describieron los aneurismas de la región de la arteria oftálmica como poco frecuentes y con particularidades muy específicas. La incidencia general de estas lesiones en otros estudios ${ }^{15,37,51,62}$ fue del 0.5 al $11 \%$, lo cual coincide con nuestros resultados.

La edad promedio de nuestra serie fue de 52 años; pero otros autores ${ }^{13,15,25,37,51,62}$ han encontrado promedios más elevados. Kakizawa et $\mathrm{a}^{13}$ encontraron un promedio de 62 años y Orz et al ${ }^{47}$ de 63 años. El promedio de edad de nuestros pacientes fue más bajo, aunque esta serie incluyó también un $16 \%$ de mayores de 60 años, una proporción adecuada si se conoce que el $15 \%$ de nuestra población es mayor de esa edad.

Hace algunos años la cirugía de los aneurismas intracraneales tenía límites de edades muy estrechos. El incremento de la expectativa de vida, el envejecimiento poblacional y un mejor estado de salud de la población determinan que los pacientes con buen estado clínico y posibilidad de actividad independiente después de la cirugía, puedan enfrentar la intervención quirúrgica satisfactoriamente, hasta que nuevos avances científico-técnicos, y nuevos métodos quirúrgicos mínimamente invasivos ayuden a obtener mejores resultados $\mathrm{s}^{6,58}$.

La existencia de una elevada frecuencia de pacientes del sexo femenino ( $80 \%$ ), y de sacos gigantes $(20 \%)$ coincide también con los hallazgos de otros estudios ${ }^{15,37,51,62}$.

Otros autores señalan que los aneurismas de la región de la arteria oftálmica pueden ser bilaterales o gemelos en el 20 al 47\% de los aneurismas múltiples ${ }^{7,22,23}$. Drake et al ${ }^{13}$ encontraron un $25 \%$, Day un $17.5 \%^{7}$ y nosotros un $12 \%$.

Hemos reportamos con anterioridad una multiplicidad general del $20 \%$ en los pacientes con aneurismas intracraneales en nuestra población ${ }^{59,60}$ y una frecuencia de 2.2 lesiones por paciente; pero la mayoría de los auto$\operatorname{res}^{7,22,23,37,51,62}$ señalan que esa frecuencia se eleva en los aneurismas de la región de la oftálmica, como ocurrió en el presente trabajo, donde la frecuencia general de multiplicidad se elevó al $60 \%$ y a un promedio de 2.4 sacos por paciente.

Los aneurismas saculares asociados a las lesiones de la arteria oftálmica fueron más frecuentes en la región de la comunicante posterior, comunicante anterior y en la región de la oftálmica contralateral. Este comportamiento por localizaciones es similar al de otras series generales de aneurismas y a la nuestra ${ }^{2,59,60}$.

La proximidad anatómica de todas estas lesiones y su ubicación en corredores anatómicos adyacentes facilita el clipaje de todas en un solo proceder quirúrgico. Esto último ha sido motivo de análisis por varios autores ${ }^{27,40}$ dedicados a este tipo de cirugía.

El abordaje pterional con algunas modificaciones meno- 


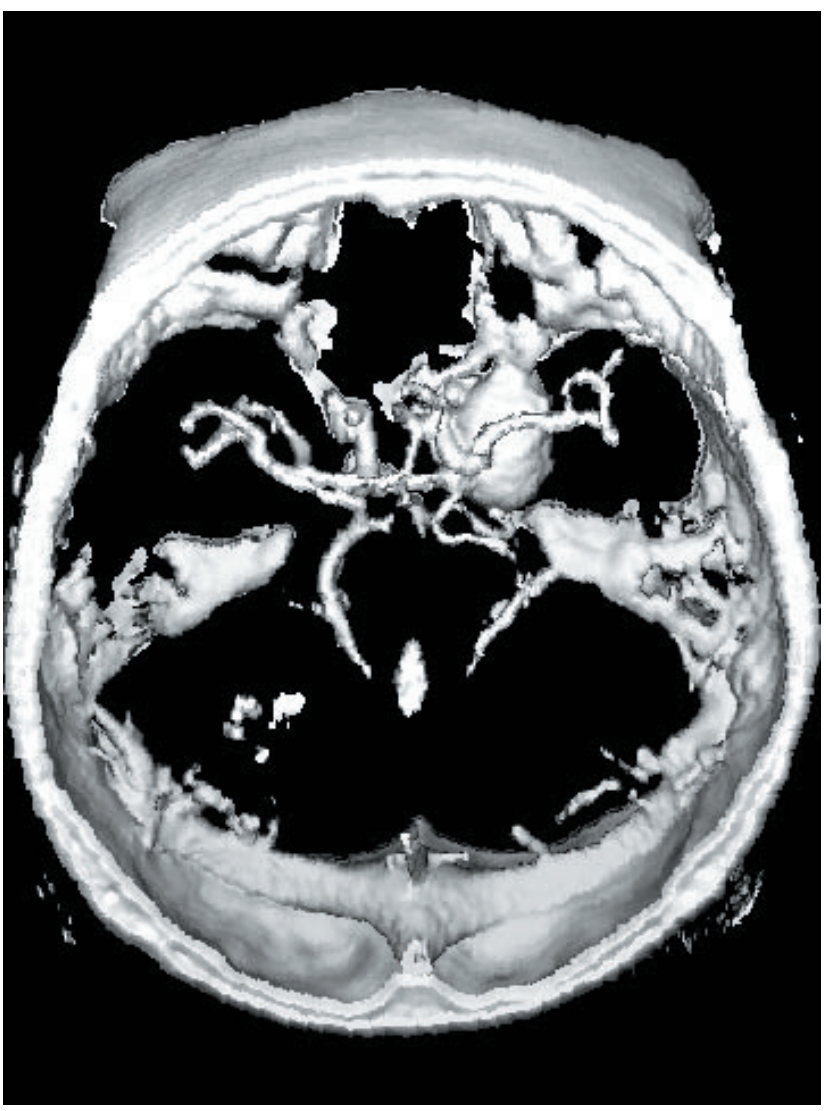

Figura 2. Angiotomografía cerebral helicoidal con reconstrucción ósea. Se observa un aneurisma gigante de la carótida en la región de la arteria oftálmica izquierda y sus relaciones con las estructuras óseas adyacentes. Esto permitió planificar el grado de resección ósea intraoperatoria. Observamos un aneurisma pequeño contralateral de igual localización.

res ha sido aceptado universalmente para el tratamiento quirúrgico de la mayoría de los aneurismas intracraneales y en el caso de los aneurismas de la región de la oftálmica es aún mayor su aceptación ${ }^{2,59,60,62}$

La diferencia básica entre el abordaje pterional para los aneurismas de la oftálmica y el utilizado en otras localizaciones está en el grado de resección ósea de la apófisis clinoides anterior, el agujero óptico, tubérculo selar y techo orbitario. La resección clinoidea es la más importante y característica. Recientemente, Giannota ${ }^{18}$ publicó una descripción detallada de esta técnica con fines educacionales y recomendó la resección clinoidea intradural para evitar la lesión del saco aneurismático.

Las resecciones óseas preconcebidas pueden resultar inadecuadas o insuficientes. Una de las ventajas de la angiotomografía cerebral helicoidal ${ }^{38}$ ha sido planificar con más exactitud esas resecciones (Figura 2). Nosotros eliminamos el ala menor del esfenoides y la porción más externa del proceso clinoideo anterior por vía extradural y completamos la resección ósea por vía intradural, según las necesidades. No hemos observado lesiones del saco procediendo de esta forma.

La resección clinoidea es muy útil para movilizar el nervio óptico, obtener el control proximal del vaso madre, identificar adecuadamente la arteria oftálmica, evitar la retracción de la vía óptica, facilitar la colocación de un clip que abarque todo el cuello aneurismático e impedir la apertura del seno cavernoso; pero en algunos sacos muy pequeños y situados dorsalmente no es indispensable una gran resección ósea.

El fresado intracraneal de las estructuras óseas adyacentes al aneurisma requiere de un entrenamiento previo adecuado. El proceso clinoideo en ocasiones puede ser una prolongación de los senos aéreos perinasales y estar en comunicación con ellos. En estos casos, tiene mucha utilidad la apertura en forma de cruz de la duramadre que lo recubre y luego coagular los bordes para poder sellar esa brecha y evitar una fístula de líquido cefalorraquídeo.

El abordaje microquirúrgico contralateral de los aneurismas múltiples ha sido reportado por varios autores $^{5,17,48,56}$. Está demostrado que la mortalidad de los aneurismas adicionales no tratados es elevada y esto puede evitarse si se logran obliterar todos los aneurismas conocidos en un solo proceder quirúrgico. En los pacientes con aneurismas de la arteria oftálmica, dada su elevada frecuencia de multiplicidad, diferentes autores ${ }^{33,47,59,60}$ han demostrado buenos resultados después de clipar varios aneurismas de diferentes localizaciones empleando una craneotomía y el abordaje simultáneo de las lesiones homo y contralaterales. Un grupo muy reducido de estos pacientes puede requerir un segundo abordaje.

En esta serie nosotros tratamos en un tiempo quirúrgico al $84 \%$ de los pacientes con aneurismas múltiples y esa cifra es similar a la reportada por otros autores ${ }^{47,48,56}$. Las topografías que motivaron una segunda intervención fueron la región de la comunicante posterior y la cerebral media contralateral. Gibo et $\mathrm{al}^{20}$ describieron cómo la mayoría de los aneurismas de la comunicante posterior se proyectan lateral o posteriormente con respecto a la arteria madre y el origen de la arteria comunicante posterior contralateral puede visualizarse sólo desde el otro lado, debido a su origen medial o posteromedial. La proporción de aneurismas de esta localización clipados contralateralmente en nuestra serie fue elevada en comparación con $\operatorname{otras}^{33,47,59,60}$.

En los aneurismas situados en la arteria cerebral media contralateral, cuando el segmento M1 es mayor de $11 \mathrm{~mm}$, se requiere de mayor retracción y buena relajación del cerebro para alcanzar el cuello aneurismático ${ }^{17,48,60}$.

Los abordajes contralaterales requieren de entrenamiento del cirujano, óptima relajación cerebral, manejo 
neuroanestésico especializado, condiciones anatómicas favorables y análisis particular de las características de cada lesión. Los pacientes con pobre relajación del encéfalo, microanatomía compleja, lesiones aneurismáticas contralaterales grandes o gigantes o cuyo fundus se localiza próximo al cirujano ofrecen mayores dificultades ${ }^{5,51,54}$.

Nosotros encontramos seis pacientes con aneurismas carótido-oftálmicos bilaterales. Las lesiones contralaterales fueron asintomáticas, pequeñas, mediales y no estaban asociadas a quiasma prefijado, por lo que facilitaron su tratamiento en un solo tiempo quirúrgico.

Los criterios que nosotros utilizamos para seleccionar el abordaje contralateral a las lesiones aneurismáticas de la región de la oftálmica bilateral fueron similares a los utilizados por otros autores ${ }^{25,51,62}$.

Los nuevos estudios microanatómicos y las mejores condiciones para el tratamiento microquirúrgico han renovado notablemente el interés por el abordaje contralateral de los aneurismas de la arteria oftálmica. En los primeros análisis, este abordaje estaba indicado en las lesiones pequeñas y mediales, aneurismas grandes no rotos que se proyectan superomedialmente, aneurismas asociados a adenomas hipofisarios y en los aneurismas que desplazan el nervio óptico superolateralmente.

El abordaje contralateral debe estar contraindicado en los aneurismas de la arteria oftálmica grandes y gigantes, en los que se originan lateralmente o desplazan superomedialmente el nervio óptico y en los pacientes con quiasma óptico prefijado.

Estudios recientes ${ }^{5,33}$ han utilizado diferentes puntos de referencias anatómicos para seleccionar el abordaje contralateral, aún en lesiones grandes. Las referencias utilizadas por esos autores fueron: las características anatómicas de la vía óptica (largo y desplazamiento de los nervios ópticos y posición del quiasma), evaluación de las estructuras adyacentes (procesos clinoideos anteriores, tubérculo selar, seno cavernoso y anillo dural) y estado de los vasos adyacentes (arteria carótida interna, arteria oftálmica e hipofisaria superior).

Kakizawa et $\mathrm{al}^{33}$ utilizaron seis parámetros para obtener una información preoperatoria máxima en la principal área de trabajo quirúrgico y así reducir los riesgos del abordaje contralateral. Emplearon tomografía computarizada tridimensional, imágenes por resonancia magnética, angiorresonancia y angiografía convencional. Los parámetros utilizados fueron: el espacio prequiasmático, la interrelación entre los nervios ópticos y la arteria carótida interna, el tamaño del cuello aneurismático, la dirección del saco y el estado del anillo dural.

Otra ventaja señalada del abordaje contralateral de los aneurismas de la región de la arteria oftálmica ha sido un menor tiempo quirúrgico, ya que no es necesaria la resección de los procesos clinoideo anterior ni la apertura del anillo dural distal que determina cierto grado de sangrado del seno cavernoso.

Las desventajas del abordaje contralateral incluyen las limitaciones en el control del vaso madre, un principio elemental en la cirugía aneurismática y la proximidad del fundus del aneurisma. Kakizawa et $\mathrm{al}^{33}$ para evitar estos inconvenientes disecan la carótida cervical contralateral antes de realizar la craneotomía y proponen otras alternativas de control como colocar un balón en la carótida interna cervical.

Nosotros no utilizamos el abordaje contralateral para todas las lesiones unilaterales y sólo lo utilizamos en lesiones bilaterales con condiciones anatómicas de disección y clipaje muy favorables.

Dolenc $^{9,10,11}$ describió el abordaje microquirúrgico craneorbitario epi-subdural para los aneurismas de la región de la oftálmica, muy similar al que utiliza en los aneurismas intracavernosos. Este abordaje no ha sido aceptado por la generalidad de los autores ${ }^{7,25}$ quienes lo consideran muy arriesgado.

Los límites microanatómicos del segmento oftálmico de la carótida intracraneal están muy bien establecidos ${ }^{25}$; pero algunos aneurismas en su crecimiento pueden extenderse hacia otros segmentos vecinos: la región carótidocava y muy por debajo del anillo, adoptando una posición extradural. En estos casos, la técnica de Dolenc ${ }^{9,10,11}$ podría evaluarse como una vía favorable para exponer todo el saco y tener un control más proximal del vaso madre. Nosotros no encontramos ningún paciente con esas características en nuestra serie.

Ferguson y Drake ${ }^{15}$ hallaron un $32 \%$ de aneurismas gigantes en esta localización y Day $^{7}$ describió un 45\%. Otros autores ${ }^{7,27,46}$ coinciden en la elevada frecuencia de aneurismas gigantes en este segmento. Nosotros encontramos un $20 \%$ de sacos gigantes, una cifra elevada, si se tiene en cuenta que la frecuencia general de aneurismas gigantes en nuestra institución fue del $4 \% 0^{57}$.

La ubicación de estos aneurismas muy próximos a la base craneal y el estado anatómico de sus paredes hacen mucho más complejo su tratamiento microquirúrgico, por eso, nosotros utilizamos siempre la técnica de descompresión-succión retrógrada descrita por Batjer ${ }^{3,4}$ y modificada por nosotros ${ }^{61}$ en los aneurismas grandes y gigantes de la región de la oftálmica.

Esta técnica permitió reducir transitoriamente el volumen del saco, realizar una disección más segura del cuello aneurismático, separarlo de las estructuras neurales, en especial, de la vía óptica y efectuar un clipaje más efectivo. Las modificaciones que nosotros realizamos de esta técnica son: primero, la utilización de la hipotermia ligera, exponiendo deliberadamente al paciente a la temperatura ambiente del salón, con lo que se logra una temperatura esofágica de 34 y $35^{\circ} \mathrm{C}$; segundo, la irrigación y aspiración 
continua del segmento arterial atrapado utilizando una solución de suero fisiológico con bajas dosis de heparina, para evitar la formación de émbolos y tercero: la selección por el neurocirujano de los lugares proximales y distales de clipaje transitorio para evitar nuevos daños vasculares (disecciones arteriales, fragmentación de placas de ateromas u oclusiones postoperatorias).

La técnica de descompresión succión retrógrada con estas modificaciones fue muy efectiva en la reducción del volumen del saco y facilitó la disección con una mayor protección de la vía visual.

En los aneurismas grandes y gigantes de esta localización se han propuesto otros procederes auxiliares para descomprimir o disminuir la tensión del saco ${ }^{16,46}$ además de la técnica de descompresión succión; pero este último y el clipaje transitorio de la carótida interna en su segmento cervical, han sido los más efectivos. Otros procederes utilizados por otros autores fueron el clipaje de la carótida intracavernosa y carótido-cava ${ }^{9,11}$ junto a las oclusiones transitorias de las arterias comunicante posterior y cerebral anterior $^{33,47}$.

Nosotros siempre disecamos y exponemos completamente la carótida cervical y sus dos ramas terminales, y si es necesario realizamos la técnica de descompresión succión retrógrada completa o de lo contrario sólo utilizamos el clipaje transitorio. Ambos procederes no se excluyen. Giannota $^{18}$ propone utilizar además, un balón intracarotídeo para ocluir la arteria madre.

La oclusión temporal de la carótida en el segmento carótido-cava, preferida por algunos autores por su proximidad al segmento oftálmico $9,11,16,46$, tiene muchos inconvenientes que analizamos detalladamente en el Cuadro 1.

Dos complicaciones frecuentes del tratamiento microquirúrgico de los aneurismas grandes y gigantes de la región de la oftálmica son el deslizamiento del clip y la oclusión del vaso madre. Estas complicaciones se evitan con mejores condiciones de clipaje y colocando las ramas del clip paralelas al vaso madre; pero la utilización de la angiografía intraoperatoria al final del proceso, permite conocer la localización exacta del clip y el estado anatómico de la arteria madre, otra ventaja más de la técnica de descompresión succión retrógrada.

Una de nuestras modificaciones a la técnica de Batjer fue la utilización de una solución de heparina que irriga continuamente el área de atrapamiento vascular. Este área está sometida a un doble sistema de irrigación-aspiración, que evita la formación de coágulos en ese segmento. La utilización de anticoagulantes a bajas dosis no estuvo relacionada con sangrados intraoperatorios ni con hematomas postoperatorios. La dosis de heparina que utilizamos fue de 5000 Uds. por litro de suero fisiológico y esa dosis no modificó el tiempo de coagulación del paciente.

Recientemente, Fan et $\mathrm{al}^{14}$ han propuesto una nueva

\section{Cuadro 1 \\ Inconvenientes de la oclusión transitoria en el área carótido-cava}

1. Requiere de la apertura del seno cavernoso.

2. Maniobra difícil en aneurismas gigantes.

3. Mayor riesgo de daño de la vía óptica.

4. La pared carotídea es más fina y débil en ese segmento.

5. Requiere un mayor entrenamiento microquirúrgico.

6. Requiere de resección ósea adicional.

7. Mayor consumo de tiempo.

8. Aprendizaje más difícil.

9. Excluye la posibilidad de la técnica de descompresiónsucción retrógrada.

modificación de la técnica de descompresión succión retrógrada en los aneurismas paraclinoideos. Estos autores introducen el angiocatéter por la arteria carótida externa y con ello evitan las disecciones de la arteria carótida interna u otros daños vasculares innecesarios. Nosotros opinamos que esa modificación complica innecesariamente el proceder auxiliar, porque la angulación del angiocatéter, para llegar hasta la luz de la carótida interna, limita su movilidad y puede ser riesgoso.

Giannota $^{18}$ considera que la forma más efectiva de realizar la técnica de descompresión succión es con un colega experimentado en métodos endovasculares. Nosotros opinamos que los neurocirujanos tienen más experiencia para identificar las modificaciones anatómicas externas en la pared arterial normal o patológica y están más preparados para realizar esta técnica.

Los déficit motores transitorios y los trastornos neuropsíquicos aparecieron en un $38 \%$ de los pacientes operados; pero en los pacientes con aneurismas grandes y gigantes, donde se realizó la técnica de descompresiónsucción retrógrada, este déficit se elevó al 50\%. El 50\% de todos ellos remitieron antes de los seis meses después de la intervención. Esta complicación se ha relacionado con diversos fenómenos tales como: retracción cerebral, contusiones y pequeñas laceraciones, edema postoperatorio, desplazamientos cerebrales, cambios hemodinámicos, torsión postclipaje y sobretodo con el enderezamiento del vaso madre después de eliminar el saco gigante ${ }^{3,52,61}$.

Estas complicaciones son más frecuentes en los propios trabajos de Batjer et al. y otros ${ }^{3,4,41}$ lo cual sugiere que las modificaciones introducidas por nosotros tienen un efecto significativo sobre ellas.

Nosotros siempre revisamos con detenimiento el estado final de los clips y de la arteria madre. El uso conveniente 
de pequeñas almohadillas de músculo, grasa, aponeurosis o poliuretano debidamente colocados evitó el movimiento de los grandes clips que utilizamos en estos sacos voluminosos, que pueden desplazarse lateralmente y ocluir parcialmente el tronco principal de la arteria madre.

Los trastornos visuales fueron frecuentes en este grupo de pacientes y hubo mejoría notable de ellos después de la intervención quirúrgica en el $64 \%$. No hubo agravamientos visuales postoperatorios, ni pacientes ciegos después de la intervención. Ambos hechos han sido reportados en otras series $^{3,7,15,31,45,50}$. Nosotros relacionamos nuestros resultados con dos hechos: Primero, el especial cuidado en no retraer las estructuras de la vía visual dañada, ni colocar retractores para desplazar las estructuras ópticas. Segundo, la utilización de la técnica de descompresión-succión retrógrada que permitió una disección del saco con menos turgencia y presión sobre la vía óptica isquémica ${ }^{3,5,36}$.

La mejoría postoperatoria de la visión fue gradual y progresiva, lo que debe tenerse en cuenta en la evolución clínica del paciente y en las explicaciones a los familiares sobre el pronóstico.

Dolenc $^{10,11}$ y otros autores ${ }^{7,41}$ que no utilizan la técnica de descompresión succión retrógrada han obtenido un menor índice de mejoría de los trastornos visuales.

Resultó lamentable que un subgrupo de pacientes con manifestaciones visuales previas y sangrado subaracnoideo posterior fueron evaluados con anterioridad por varios especialistas que atribuyeron sus déficit visuales a problemas inherentes al envejecimiento, presbicia, neuropatía óptica isquémica, glaucoma, catarata u otros procesos.

Los aneurismas son lesiones potencialmente letales en pacientes de la sexta y séptima década de la vida y requieren de un alto índice de sospecha clínica. El diagnóstico precoz puede orientar el estudio imaginológico y detectar la lesión antes del sangrado ${ }^{29,44}$.

El sacrificio de algunas ramas perforantes del segmento oftálmico que irrigan el parénquima cerebral y la región pituitaria durante la disección y el clipaje de los aneurismas no determinan trastornos endocrinos, porque estas estructuras son irrigadas por diversas arterias. Gibo et $\mathrm{al}^{19}$ en un estudio microanatómico de la región evidenciaron que estas estructuras reciben nutrición de las arterias hipofisaria superior, hipofisaria inferior, comunicante posterior y ramas perforantes directas de la carótida interna. La causa principal de la diabetes insípida es la distorsión del tallo hipofisario durante la disección del aneurisma y los fenómenos de enderezamiento pituitario postoperatorio. Esto explica la baja incidencia de trastornos endocrinos en nuestra serie, su elevado índice de recuperación y la inexistencia de déficit hormonales de la anterohipófisis seis meses después de la intervención. Los trastornos endocrinos nunca fueron permanentes en nuestra serie y este déficit debe evaluarse con optimismo con el paciente y la familia.
Aunque el tratamiento ideal de los aneurismas es el clipaje directo del cuello, existen condiciones particulares como el tamaño del cuello, la accesibilidad, el tamaño de la lesión, el grosor de la pared y otras dificultades técnicas que entorpecen este procedimiento en la región paraclinoidea. Métodos alternativos de tratamiento como la oclusión proximal del vaso madre, y el atrapamiento y la escisión del aneurisma son inferiores al clipaje directo, porque sacrifica la anatomía normal, determinan una afectación hemodinámica y requieren de técnicas de revascularización para mantener el flujo sanguíneo en el territorio involucrado y prevenir complicaciones isquémicas definitivas ${ }^{61}$.

Nosotros no utilizamos derivaciones sanguíneas; pero uno de nuestros pacientes tenía un aneurisma gigante muy voluminoso, parcialmente trombosado y decidimos abrir el saco, resecar la pared lesionada y reconstruirla. El uso de las derivaciones sanguíneas está indicada cuando la circulación colateral del cerebro es insuficiente ${ }^{30}$.

El método de tratamiento endovascular con espirales trombogénicos dentro del aneurisma ha demostrado su eficacia terapéutica, pero en caso de sacos grandes o gigantes, este método tiene algunas limitaciones y su eficacia se muestra moderada por oclusiones incompletas y por la limitación del método para eliminar completamente la compresión de la vía óptica. Los aneurismas seleccionados para método endovascular deben tener un cuello menor de cinco milímetros y un índice cuello-domo adecuado; pero tales condiciones anatómicas son muy poco frecuentes en esta localización ${ }^{28,39,53}$.

Las dificultades para lograr el empaquetamiento denso de los espirales dentro de los sacos grandes y gigantes, junto con la frecuencia de un cuello ancho, hacen que la mayoría de los cirujanos prefieran los métodos quirúrgicos.

Lanzino et $\mathrm{al}^{39}$, Grubet et $\mathrm{al}^{21}$, y otros ${ }^{24,54}$ utilizan espirales trombogénicos en pacientes con aneurismas de la arteria oftálmica en mal estado clínico neurológico, edad avanzada, con enfermedades intercurrentes graves que contraindican la cirugía.

Otros autores han utilizados métodos combinados microquirúrgicos y endovasculares; pero reportan índices elevados de isquemia postoperatoria y oclusión incompleta de los sacos en el $56 \%$ de los pacientes ${ }^{1,26,43}$.

Nagasawara et $\mathrm{al}^{41}$ compararon los métodos endovasculares y microneuroquirúrgicos de tratamiento de los aneurismas de la región de la oftálmica y sugieren los métodos endovasculares en las siguientes condiciones técnicas: el segmento $\mathrm{C} 3$ se extiende dentro del proceso clinoideo, cuello aneurismático calcificado o ateromatoso y fuertes adherencias del domo al anillo dural. Como estas condiciones sólo se pueden reconocer durante la cirugía, los grupos combinados de tratamiento microquirúrgico y endovascular parecen ser la mejor opción terapéutica en la 
actualidad.

Los aneurismas de la región de la arteria oftálmica son lesiones poco frecuentes, pero con características muy peculiares. Son más usuales en mujeres, tienen una elevada frecuencia de multiplicidad y de aneurismas gigantes. La íntima relación de estos aneurismas con la vía óptica y la vecindad con las estructuras óseas de la base craneal y el seno cavernoso determinan notables dificultades y complejidades técnicas en el quirófano. El tratamiento microquirúrgico permite clipar en un solo proceder quirúrgico lesiones múltiples uni o bilaterales. Los aneurismas gigantes de esta localización requieren de técnicas especiales para reducir el tamaño del saco y disminuir la tensión de sus paredes. Esto facilita la disección y clipaje del cuello con poca morbilidad. Los déficit motores y las manifestaciones neuropsíquicas postoperatorias desaparecen varios meses después de la intervención. Los déficit visuales, muy frecuentes en este tipo de aneurismas, permiten hacer un diagnóstico precoz y la cirugía mejora mucho estos trastornos, siempre que se traten precozmente. El estudio adecuado de los problemas visuales en los pacientes de la sexta y séptima década de la vida ayudan a realizar un diagnóstico precoz de muchas de estas letales e ignoradas lesiones.

\section{Bibliografía}

1. Amautovic, K.L., Al-Mefty, O., Angtuaco, E.: A combined microsurgical skull-base and endovascular approach to giant and large paraclinoid aneurysms. Surg Neurol 1998; 50: 504- 523.

2. Armonda, R.A., Nehls, D.G.: Multiple intracranial aneurysms. En Carter LP, Spetzler RF. Neurovascular Surgery. New York. Ed. Mc Graw Hill Inc. 1995 p. 807-814.

3. Batjer, H.H., Kopitnik, T.A., Giller, Ca., Samson, D.S.: Surgery of paraclinoid carotid artery aneurysms, J Neurosurg 1994; 80: 650-658.

4. Batjer, H.H., Samson, D.S.: Retrograde decompression of giant paraclinoidal aneurysms. Technical notes, J Neurosurg 1990; 73:305-306.

5. Cawley, C.M., Zipfel, G.J., Day, AL.: Surgical treatment of paraclinoidal and ophthalmic aneurysms. Neurosurg Clin N AM 1998; 9: 765-783.

6. Chung, R.J., Carter, B.B., Norbasa, A., Budzick, R., Putman, C., Ogilvy, C.S.: Management, outcomes for ruptured and unruptured aneurysms in the elderly. Neurosurgery 2000; 47: 827-833.

7. Day, A.L.: Aneurysms of the ophthalmic segment. A clinical and anatomical analysis. J Neurosurg 1990; 72: 677691.

8. De Jesús, O., Sekhar, J.N., Riedel, C.J.: Clinoid and paraclinoid aneurysms. Surgical, anatomy, operative techniques and outcome. Surg Neurol 1999; 51: 477-488.
9. Dolenc, V.V.: A combined epi and subdural direct approach to carotid-ophthalmic artery aneurysms. J Neurosurgery 1985; 62: 667-672.

10. Dolenc, V.V.: A combined transorbital-transclinoid and transsilvian approach to carotid-ophthalmic aneurysms without retraction of the brain. Acta Neurochir Suppl (Wien) 1999; 72: 89-97.

11. Dolenc, V.V.: Carotid-ophthalmic aneurysms. En Carter, L.P., Spetzler, R.F. Neurovascular Surgery. New York. Ed. Mac Graw Hill Inc. 1995 p 673-686.

12. Drake, C.G., Hunt, W.E., Sano, K., Kasell, N., Teasdale, G., Pertuiset, B. et al.: Report of the World Federation of Neurological Surgeons. Committee on a universal subarachnoid grading scale. J Neurosurg 1988; 68: 986-987.

13. Drake, C.G., Vanderlinden, R.G., Amacher, A.L.: Carotid-ophthalmic aneurisms. J Neurosurg 1968; 29: 24-31.

14. Fan, Y.W., Chan, K.H., Lui, W.M., Hung, K.N.: Retrograde suction decompression of paraclinoid aneurysm-a revised technique. Surg Neurol 1999; 51: 129-131.

15. Ferguson, G.G., Drake, C.G.: Carotid-Ophthalmic aneurysms: Visual abnormalities in 32 patients and the results of treatment. Surgical Neurology 1981; 16: 1-8.

16. Flamm, E.S.: Suction decompression of aneurysms. Technical note. J Neurosurg 1981; 54: 275-276.

17. Fries, G., Perneczky, A., Van Lindert, E., BahadoriMortasawi, F.: Contralateral and ipsilateral microsurgical approaches to carotid-ophthalmic aneurysms. Neurosurgery 1997, 41: 333-343.

18. Giannota, S.L.: Ophthalmic segment aneurysms surgery. Neurosurgery 2002; 50: 558-562.

19. Gibo, H., Kobayashi, S., Kyoshima, K., Hokama, M.: Microsurgical anatomy of the arteries of the pituitary stalk and gland as viewed from above. Acta Neurochir (Wien) 1988; 90: 60-66.

20. Gibo, H., Carter, C.C., Rhoton, A.L. Jr.: Microsurgical anatomy of the supraclinoid portion of the internal carotid artery. J Neurosurg 1981; 55: 560-574.

21. Gruber, A., Killer, M., Bavinzski, G., Richling, B.: Clinical and angiographic results of endosaccular coiling treatment of giant and very large intracranial aneurysms. A seven year, single-center experience. Neurosurgery 1999; 45: 793-804.

22. Guidetti, G., La Torre, E.: Carotid ophthalmic aneurysms. A series of 16 cases treated by direct approach. Acta Neurochir (Wien) 1970; 22: 289-304.

23. Guidetti, G., La Torre, E.: Management of Carotidophthalmic aneurysms. J Neurosurg 1975; 42: 438-442.

24. Gurian, J.H., Viñuelas, F., Gugleilmi, G., Gobin, Y.P., Duckwiler, G.R.: Endovascular embolization of superior hypophyseal artery aneurysm. Neurosurgery 1996; 39: 11501154.

25. Hoh, B.L., Carter, B.S., Budzik, R.F., Putman, C.M., Ogilvy, C.S.: Results After surgical and endovascular treat- 
ment of paraclinoid aneuryms by a combined neurovascular team. Neurosurgery 2001; 48: 78-90.

26. Hoh, B.L., Cheung, A.C., Rabinov, J.D., Pryor, J.C., Carter, B.S., Ogilvy, C.S.: Results of a Prospective Protocol of Computed Tomographic Angiography in place of catheter angiography as the diagnostic and pretreatment planning study for cerebral aneurysms by a combined neurovascular team. Neurosurgery 2004; 54: 1329-1342.

27. Hongo, K., Watanabe, N., Matsushima, N., Kobayashi, S.: Contralateral pterional approach to giant internal carotidophthalmic aneurysms: technical case report. Neurosurgery 2001; 48: 955-959.

28. Hopkins, N.L., Lanzino, G., Guterman, L.R.: Treating complex nervous system vascular disorders though a needle stick: Origins, evolution and future of neuroendovascular therapy Neurosurgery 2001; 48: 463-475.

29. Ito, K., Hongo, K., Kakizawa, Y., Kobayashi, S.: Three-dimensional contrast medium-enhanced computed tomographic cisternography for preoperative evaluation of surgical anatomy of intradural paraclinoid aneurysms of the internal carotid artery: technical note. Neurosurgery 2002; 51 : 1089-1092.

30. Jafar, J.J., Russell, S.M., Woo, H.H.: Treatment of giant intracranial aneurysms with saphenous vein extracranial-to intracranial bypass grafting: indications, operative technique and results in 29 patients. Neurosurgery 2002; 51: 138-146.

31. Jea, A., Baskaya, M.K., Morcos, J.J.: Penetration of the optic nerve by an internal carotid artery- ophthalmic artery aneurysms: Case report and literature review. Neurosurgery 2003; 53: 996-1000.

32. Jennett, B., Bord, M.R.: Assesment of outcome after severe brain damages. A practical scale. Lancet 1975; 1: 480484.

33. Kakizawa, Y., Tanaka, Y., Orz, Y., Iwashita, T., Hongo, K., Kobayashi, S.: Parameters for contralateral approach to ophthalmic segment aneurysms of the internal carotid artery. Neurosurgery 2000; 47: 1130-1136.

34. Kassell, N.F., Torner, J.C., Haley, C., Jane, J.A., Adams, H.P., Kongable, G.L.: The International Cooperative Study on the timing of Aneurysms Surgery. Part 1: Overall management results. J Neurosurg 1990; 73:18-36.

35. Kassell, N.F., Torner, J.C., Jane, J.A., Haley, E.C., Adams, H.P.: The International Cooperative study on the timing of aneurysms surgery Part 2: Surgical results. J Neurosurg 1990; 73: 37-47.

36. Kinouchi, H., Mizoi, K., Nagamine, Y., Yanagida, N., Mikagua, S., Suzuki, A., et al.: Anterior paraclinoid aneurysms. J Neurosurg 2002; 96: 1000-1005.

37. Kyoi,, K., Gega, A., Tada, T., Iida, N., Tsunoda, S., Utsumi, S., et al.: Carotid-ophthalmic aneurysms and visual abnormalities. Shinkei Geka 1986; 14: 1197-1204.

38. Kawashima, M., Matsushima, T., Miyazono, M., Hirokawa, E., Baba, H.: Two surgical cases of internal carotid- ophthalmic artery aneurysms: special reference to the threedimensional CT angiography. Neurol Res 2002; 24: 825-828.

39. Lanzino, G., Wakloo, A.K., Fessler, R.D., Hartney, M.L., Guterman, L.R., Hopkins, L.N.: Efficacy and current limitations of intravascular stents for intracranial internal carotid, vertebral and basilar artery aneurysms. J Neurosurg 1999; 91: 538-546.

40. Mc Mahon, J.H., Morgan, M.K., Dexter, M.A.: The surgical management of contralateral anterior circulation intracranial aneurysms. J Clin Neurosci 2001; 8: 319-324.

41. Nagasawa, S., Kawabata, S., Deguchi, J., Kuroiwa, T., Ohta, T., Tsuda, E.: Microsurgical results of paraclinoid aneurysms of the internal carotid artery: microsurgery versus intravascular surgery. No Shinkei Geka 1999; 27 : 809-816.

42. Nehls, D.G., Flom, R.A., Carter, L.P., Spetzler, R.F.: Multiple intracranial aneurysms: determining the site of rupture. J Neurosurg 1985; 63: 342-348.

43. Ng, P.Y., Huddle, D., Gunel, M., Awad, I.A.: Intraoperative endovascular treatment as an adjunct to microsurgical clipping of paraclinoid aneurysms. J Neurosurg 2000; 93: 554560 .

44. Nishihara, M., Tamaki, N.: Usefulness of volume-rendered three-dimensional computed tomographic angiography for surgical planning in treating unruptured paraclinoid internal carotid artery aneurysms. Kobe J Med Sci 2001; 47: 221230 .

45. Norwood, E.G., Kline, L.B., Chandra-Sekar, B.. Harsh, G.R. $3^{\text {rd }} .:$ Aneurysmal compression of the anterior visual pathways. Neurology 1986; 36: 1035-1041.

46. Ogilvy, C.S.: Paraclinoid carotid aneurysms. In Ojeman, R.G., Ogilvy, C.S., Cromwell, R.M., Heros, R.C., eds. Surgical management of neurovascular diseases. 3 ed. Baltimore: Williams and Wilkins; 1995, p. 185-213.

47. Orz, Y., Osawa, M.. Tanaka, K., Kyoshima, K., Kobayashi, S.: Surgical outcome for multiple intracranial aneurysms. Acta Neurochir (Wien) 1996; 138: 411-417.

48. Oshiro, E.M., Rini, D.A., Tamargo, R.J.: Contralateral aproaches to bilateral cerebral aneurysms. A microsurgical anatomical study. J Neurosurg 1997; 87: 163-169.

49. Rhoton, A.L. Jr.: Aneurysms. Neurosurgery 2002, 51 (4 Suppl) S 121-158.

50. Ringel, R.A., Brick, J.F.: Carotid-ophthalmic aneurysm masquerading as optic neuritis. Neurol Neurosurg Psychiatry 1986; 49: 460-464.

51. Sengupta, R.P., Gryspeerdt, G.L., Hankinson, J.: Carotid-ophthalmic aneurysms. J Neurol Neurosurg Psychiatry 1976; 39: 837-853.

52. Tanaka, Y., Kobayashi, S.: Multiple clipping technique for a large and giant internal carotid artery aneurysms and complication. J Neurosurg 1994; 80: 635-642

53. Thornton, J., Aletich, V.A., Debrun, G.M., Alazzaz, A., Misra, M., Charbel, F., et al.: Endovascular treatment of 
paraclinoid aneurysms. Surg Neurol 2000; 54: 288-299.

54. Thornton, J., Debrun, G.M., Letish, V.A., Bashir, Q., Charbel, F.T., Ausman, J.: Follow-up angiography of intracranial aneurysms treated with endovascular placement of Guglielmi detachable coils. Neurosurgery 2002; 51: 239-250.

55. Trobe, J.D., Glaser, J.S.: The visual field manual. A practical guide to testing and interpretation. Gainesville: Triad Publishing. 1983 pp. 65-71.

56. Vajda, J., Juhász, J., Pasztor, E., Nyary, I.: Contralateral approach to bilateral and ophthalmic aneurysms. Neurosurgery 1988; 22: 662-268.

57. Vega Basulto, S.D., Silva Adán, S., Laserda Gallardo, A., Peñones Montero, R., Varela Hernández, A.: Aneurismas intracraneales supratentoriales gigantes. Neurocirugía 2003, 14: $16-24$.

58. Vega-Basulto, S.D., Silva, S., Mosquera, G., Varela, A.: La cirugía de los aneurismas intracraneales en la séptima y octava década de la vida. Neurocirugía 2002; 13: 371-377.

5 9. Vega-Basulto, S.D., Silva-Adán, S., Peñones-Montero,
R.: Aneurismas intracraneales múltiples en Camaguey. Rev Neurol 2003; 37: 112-118.

60. Vega Basulto, S.D., Silva Adán, S., Peñones Montero, R.: Tratamiento quirúrgico de los aneurismas intracraneales múltiples. Neurocirugía 2003; 14: 385-91.

61. Vega-Basulto, S.D.: Técnica de descompresión-succión retrógrada en los aneurismas paraclinoideos. Rev Neurol 2003; 37: 312-317.

62.Yasargil, M.G., Gasser, J.C., Hodosh, R.M., Rankin, T.V.: Carotid-ophthalmic aneurysms: direct microsurgical approach. Surg Neurol 1977; 8: 155-165.

Vega-Basulto, S.D.; Gutiérrez-Muñoz, F.G.; Mosquera- Betancourt, G.; Rivero-Truit, F.; Vega-Trenado, S.A.: Aneurismas de la región de la arteria oftálmica. Neurocirugía 2006; 17: 303-316.

Correspondencia postal: Sergio Diego Vega Basulto. Domingo Puentes 5. La Caridad. Camagüey 70300. Cuba 